\title{
Effectiveness of Neurofeedback Associated with Cognitive Rehabilitation Therapy on Children with Attention Defect Hyperactivity Disorder (ADHD)
}

\author{
Mohammad Kianbakht ${ }^{1}$, Sedighe Naghel ${ }^{2}$, Freshte Alidadi ${ }^{3}$, Vahid Nejati ${ }^{4}$, \\ Hossien Kohandel ${ }^{5}$, Ghasem Ansarini ${ }^{6}$, Vida Namdari ${ }^{7}$
}

\section{ABSTRACT:}

Objectives: Neurofeedback is known as a modern therapy for hyperactive children, nevertheless it is not a complete therapy for these patients. The goal of this therapy is to create a complete therapy for ADHD children, so neurofeedback is used with cognitive rehabilitation therapy to make more integrated therapy.

Method: this is an experimental study. 30 subjects were selected through random selection from women who referred to 5 clinics of Tehran city, and they were placed into 2 experimental groups and 1 control group. Groups are matched in age, sexuality and economic situation. One group just received neurofeedback into 10 sessions, another group received neurofeedback plus cognitive rehabilitation therapy at the same during, and control group received no therapy or they leaved the therapy at the first steps. Integrated visual and auditory test (IVA) was used as pretest and posttest, to measure two factors (attention and impulsivity) in ADHD children. Cognitive rehabilitation therapy protocol is based on work of Nejati (1391). Data evaluated by dependent T test and analysis of covariance.

Results: according to findings, there is meaningful difference between all groups in attention and impulsivity factors. The group who received neurofeedback plus cognitive rehabilitation therapy showed more improvement in attention and impulsivity factors. Also there was a meaningful difference between neurofeedback group and control group in attention factor.

Conclusion: neurofeedback is supposed as a facilitator therapy to improve attention factor in ADHD children, but not as a complete therapy, especially about performance factors. Also, it is better to use a complementary therapy such as cognitive rehabilitation therapy that focuses more on performance factors. It leads to more positive effects on impulsivity or even hyperactivity.

Keywords: Neurofeedback, ADHD, rehabilitation, cognitive training, IVA test.

${ }^{1} \mathrm{Ph} . \mathrm{D}$ student of clinical psychology, Department of Psychology, Aligarh Muslim University, INDIA

${ }^{2}$ M.A. of Psychology, Islamic Azad University, Central Tehran, Iran

${ }^{3}$ M.A. of General Psychology, AllameTabatabai University, Iran

${ }^{4}$ M.A. of Psychology, Islamic Azad University, Central Tehran, Iran

${ }^{5} \mathrm{Ph} . \mathrm{D}$ student of clinical psychology, Department of Psychology, Aligarh Muslim University, INDIA

${ }^{6} \mathrm{Ph} . \mathrm{D}$ student of clinical psychology, Department of Psychology,Aligarh Muslim University, INDIA

${ }^{7}$ Ph.D student of clinical psychology, Department of Psychology, Aligarh Muslim University, INDIA

(c) 2015 I M Kianbakht, S Naghel, F Alidadi, V Nejati, Hossienkohandel, Ghasemansarini, V Namdari; licensee IJIP. This is an Open Access Research distributed under the terms of the Creative Commons Attribution License (http://creativecommons.org/licenses/by/2.0), which permits unrestricted use, distribution, and reproduction in any Medium, provided the original work is properly cited. 


\section{Effectiveness of Neurofeedback Associated with Cognitive Rehabilitation Therapy on Children with Attention Defect Hyperactivity Disorder (ADHD)}

Attention deficit and Hyperactivity disorder (ADHD) is a pervasive developmental disorder which attention deficit, hyperactivity and impulsion are as the main symptoms and often includes executive function disorder, too (1-2). Clearly, this disorder can lead to many difficulties in child development process including poor academic performance, high physical and verbal aggression (3). There are different neuropsychological models in order to explain defects in self-regulation underlying these symptoms, for example deficits in the dealing out of neuropsychological resources (4), maladjustment of interacting neuronal networks (5), and reward deficiency syndrome (6). Because ADHD has serious developmental implications in the short and long term that encompass problems in deferent aspect of life including social, academic and quality of life (7) treatment should begin early, in childhood (9).

Though, general treatment such as medication or behavior therapy is considered strong and suitable treatments for ADHD children, but they still encounter fundamental limitations. Medication, as primary treatment of ADHD (8), often improves symptoms; however, it is far away from normal level and permanent, long-term medication therapy is effective about $13.2 \%$ to $64 \%(10,18)$. Once, medication therapy stopped the symptoms usually recurrent. In addition, stimulating drugs have no impact on $20-30 \%$ of children or side effects including insomnia, decreased appetite $(11,12)$ and or preventing child development will occur followed by stopping medication therapy (13). Hence, parents do not prefer medication therapy.

Some treatments with experimental evidences in ADHD treatment include parents training and behavioral interventions in school (14-15). Other interventions are promising in treatment such as academic interventions, summer treatment plan for these types of children (16) and programs that combined parents training and training social skills. However, mental treatments are not as much effective as medication therapy since it is not possible to generalize them to all contexts (17).

Learning ADHD symptoms even in classrooms, with common limitation of current treatments, provide the importance of alternative treatment options to apply at schools such as computer based attention training (CompAt). CompAt, based on brain flexibility and conditional agent theories, is designed to improve core and critical skills that commonly have defects in ADHD children. Two main types of it are Neurofeedback (NF) and Cognitive training (CT) (18).

Neurofeedback and such treatments had promising results and also with less side effects (21). Obtained findings of EEG in ADHD children showed increased Theta waves activity as well as increasing Theta ratio to Beta in the frontal cortex. So, neurofeedback is mostly applied in training how to increase Beta waves (a situation requiring attention) and decrease Theta $(\theta$ ) waves to people. Neurofeedback, with these specific brain waves patterns, provides an immediate feedback of how people concentrate. 


\section{Effectiveness of Neurofeedback Associated with Cognitive Rehabilitation Therapy on Children with Attention Defect Hyperactivity Disorder (ADHD)}

Any change in brain waves patterns on computer screen is illustrated through characters and figures moving with auditory feedback. Individuals, by practicing, learn to change their brain waves in achieving a target and enhancing attention (19). Indeed, they learn to control underlying processes of these brain waves and to improve cognitive, emotional and behavioral selfregulatory (20).

Previous studies demonstrated the effect of neurofeedback treatment on children with ADHD (21). Meisel et al (2013) in a research studied the effect of neurofeedback and medication therapy on ADHD children. They investigated the results based on parents and teachers reports. Both groups presented satisfactory results, in 6 months follow-up, in improving ADHD symptoms. However, the group received neurofeedback treatment showed more fundamental improvement in academic performance. This research is considered as the first controlled study in the next six month follow-up on ADHD with non- pharmacologic treatment and again proving the impact of neurofeedback.

Cognitive training applies frequent computer feedbacks to enhance correct responses beside training attention, working memory and reducing impulsiveness. Studies showed that cognitive interventions and effective training will improve working memory; further, it reduces the amount of disorder symptoms reported by parents and teachers (22). Practicing working memory also contains improvement in various areas including improving mathematic problem solving and or reading comprehension (23).

Gevensleben et al (2009) used neurofeedback along with cognitive training and found out that teachers and parents reported more improvements. But since the sample heterogeneity, it was not generalizable to larger population (24). In another study, Steiner et al (2014) compared neurofeedback method with cognitive training. In this study, children in neurofeedback group offered better results as compared to cognitive training and control group. The results in this study were also investigated in terms of teachers and parents reports. None of previous studies combined neurofeedback and cognitive rehabilitation and investigated the integrated impact of these two issues (2).

Studies showed that executive parts of working memory are one of the damaged functions in these people (25). There are two points of views. Barkley (2006) believed that failure in response inhibition is the main cause of this disorder which may lead to damaging working memory and other executive functions (26). In contrast, Rapport et al believed that this disorder is caused by early failure in working memory leading to executive functions' defects (25).

As it is presented, both approaches, in spite of different insights, represent working memory defects in this disorder. Working memory is a set of cognitive processes to maintain and manipulate different information of daily routines. Some of these daily routines are rehearsal, 


\section{Effectiveness of Neurofeedback Associated with Cognitive Rehabilitation Therapy on Children with Attention Defect Hyperactivity Disorder (ADHD)}

retrieval, manipulation and controlled attention (27). Hence, applying an approach that improves working memory can lead to improving the symptoms of attention deficit and hyperactivity, too. Cognitive rehabilitation is one program which can be regarded as one of the ways of improving working memory.

This program, designed by Nejati (2010), is a software program in order to enhance maintenance, transfer, updating and inhibiting information abilities. Its effectiveness on working memory was provided in research efforts. This program includes a group of organized hierarchical tasks enhancing several aspects of working memory (maintenance, transferring, and updating as well as inhibiting information). The fundamental principle of this program is as follows:

1. Tasks are hierarchically organized and getting more difficult based on user response beyond sessions.

2. Performing tasks correctly will be immediately rewarded; and rewards are gradually offered in longer delays.

3. Tasks were designed based on various functions of working memory including updating, transferring and inhibition.

4. Tasks are fun and presented with motivational stimulators so that enhance patient's motivation.

5. Tasks may be retrieved until the patient got the desired level.

6. Developing the program depends on patient efficacy; and, the therapist is required to improve task level (28).

Therefore, considering the effect of this program on cognitive memory, it is assumed that it can also be effective as an alternative, supplementary treatment in improving attention deficiency disorder. According to above, this study intended to investigate a more complete treatment in improving attention deficiency and hyperactivity that concentrate on working memory executive functions and to achieve more positive results besides neurofeedback which its effect has been proved in previous studies. Thus, neurofeedback effect with and without cognitive rehabilitation was compared. It is assumed that neurofeedback will be effective alone, but integrating it with cognitive rehabilitation and training would lead to better results.

\section{METHODS}

This study is an experimental research design with pre- and post- test with control group. So, three experimental groups were randomly selected from those referred to the clinic. First, the participants were 85 children with symptoms of attention deficit and hyperactivity selected based on DSM-V criteria. Of these, 45 individuals were randomly assigned in to three (15 individuals) groups. Subjects of one group just received neurofeedback, the other group received a combination of neurofeedback and cognitive rehabilitation and the last group, as control group, did not receive any treatments. All children were homogenized in all aspects. The age range was within 5 to 7 years old from the same economic level. Moreover, they all had no history of 


\section{Effectiveness of Neurofeedback Associated with Cognitive Rehabilitation Therapy on Children with Attention Defect Hyperactivity Disorder (ADHD)}

ADHD treatment, medication and nonmedication treatment, in order to avoid effecting on the present study results. Initially, the subjects were tested by IVA+plus to measure their impulsion and attentions' basic rate. Next, two experimental groups received ten treatment sessions according to protocol and the determined timing. Finally, a post test IVA+plus was done in order to measure changes during treatments. Cognitive training program or cognitive rehabilitation in the combined group was done one day later followed by neurofeedback.

\section{Tools}

IVA+PLUS test: this is a continuous auditory- visual 13-minute test evaluating two main factors i.e. impulsion response control and attention. IVA+PLUS test is formulated relying on statistical and diagnostic manuals of mental disorders DSM-IV which distinguishes different types ADHD including the attention deficit type, hyperactivity type (impulsion), combined type as well as unknown NOS type. Moreover, this test was used to study difficulties and other disorders such as self-inhibitory problems related to head traumas, sleep disorders, anxiety, learning disorders, dementia and other medical problems. This test was applied for children older than 6 and adults. The time required is about 20 minutes. The tasks were responding or not responding to 500 test stimuli. Each stimulus is only provided in $1.5 \mathrm{~s}$. So, the test requires attention. IVA+PLUS test consists of 6 total scales and 22 subscales classified in to 5 groups: response inhibition, attention, attribution, indicator as well as delicate movements. However, this study just considers the scores of two response and attention scales.

\section{RESULTS}

One-way between groups covariance analysis was done to compare the effectiveness of two different interventions of participants' response inhibition and attention scales. Intervention type (neurofeedback and neurofeedback with cognitive rehabilitation treatment) was independent variable and dependent variable was consisted of two subscales of IVA+PLUS test. Participants' scores of two response inhibition and attention subscales before intervention were used as covariance in this analysis. 


\section{Effectiveness of Neurofeedback Associated with Cognitive Rehabilitation Therapy on Children with Attention Defect Hyperactivity Disorder (ADHD)}

Table 1: summary of covariance for therapy effects on 2 subscales of IVA+PLUS test (compulsivity and attention) before therapy.

\begin{tabular}{|c|c|c|c|c|}
\hline Variable source & ss & df & Ms & f-ratio \\
\hline $\begin{array}{c}\text { Covariance (impulsive } \\
\text { scores before therapy) }\end{array}$ & 143.403 & 1 & 143.403 & $0.538^{* *}$ \\
\hline Main effect of therapy & 51.648 & 2 & 25.824 & $8.167^{* *}$ \\
\hline Residual error & 123.322 & 39 & 3.162 & - \\
\hline $\begin{array}{c}\text { Covariance (attention } \\
\text { scores before therapy) }\end{array}$ & 138.496 & 1 & 138.496 & $39.697 * *$ \\
\hline Main effect of therapy & 48.174 & 2 & 24.087 & $6.904^{* *}$ \\
\hline Residual error & 136.064 & 39 & 3.489 & - \\
\hline
\end{tabular}

Preliminary analyses were done to ensure that normality, linearity; variance homogeneity, regression slope homogeneity and the reliability of covariance hypotheses were not violated.

Table 2: mean and standard deviation scores in attention scale

\begin{tabular}{|c|c|c|c|}
\hline Group & mean & Standard deviation & Number of subjects \\
\hline $\begin{array}{c}\text { 1. neurofeedback \& } \\
\text { rehabilitation }\end{array}$ & 93.06 & 1.751 & 15 \\
\hline 2. neurofeedback & 75.11 & 2.892 & 15 \\
\hline 3. control & 74.85 & 14.693 & 45 \\
\hline Total & 81.10 & 12.539 & 15 \\
\hline
\end{tabular}

Table 3: mean and standard deviation scores of impulsivity scale, after therapy

\begin{tabular}{|c|c|c|c|}
\hline Group & mean & Standard deviation & Number of subjects \\
\hline $\begin{array}{c}\text { 1. neurofeedback \& } \\
\text { rehabilitation }\end{array}$ & 93.12 & 1.463 & 15 \\
\hline 2. neurofeedback & 74.21 & 2.927 & 15 \\
\hline 3. control & 74.93 & 15.763 & 45 \\
\hline Total & 80.97 & 12.505 & 15 \\
\hline
\end{tabular}


Table 4:Weighted Mean of 3 situation of experiments on impulsivity subscale

\begin{tabular}{|c|c|c|c|c|}
\hline groups & mean & Standard deviation & \multicolumn{2}{|c|}{ confidence level 95\% } \\
\cline { 3 - 5 } & & & $\begin{array}{c}\text { Low } \\
\text { limit }\end{array}$ & Up limit \\
\hline $\begin{array}{c}\text { 1. neurofeedback \& } \\
\text { rehabilitation }\end{array}$ & 91.619 & 1.410 & 88.767 & 94.472 \\
\hline 2. neurofeedback & 81.859 & 1.689 & 78.443 & 85.275 \\
\hline 3. control & 69.689 & 0.486 & 68.706 & 70.671 \\
\hline
\end{tabular}

Table 5: Weighted Mean of 3 situation of experiments on impulsivity subscale

\begin{tabular}{|c|c|c|c|c|}
\hline groups & mean & Standard deviation & \multicolumn{2}{c|}{ confidence level 95\% } \\
\cline { 4 - 5 } & & & $\begin{array}{c}\text { Low } \\
\text { limit }\end{array}$ & Up limit \\
\hline $\begin{array}{c}\text { 1. neurofeedback \& } \\
\text { rehabilitation }\end{array}$ & 91.606 & 1.521 & 88.530 & 94.682 \\
\hline 2. neurofeedback & 81.846 & 1.771 & 78.264 & 85.427 \\
\hline 3. control & 69.668 & 0.511 & 68.635 & 70.700 \\
\hline
\end{tabular}

Followed by correcting pre-intervention scores, there was seen a significant difference among three groups (two under intervention groups and one control group) response inhibition subscale, $\mathrm{f}(2,39)=8.167, \mathrm{p}=.001$, Partial Eta Squared 0.29.

Moreover, there was also seen a significant difference among three groups (two under intervention groups and one control group) in attention subscale followed by correcting preintervention scores, $\mathrm{f}(2,39)=6.904, \mathrm{p}=.003$, Partial Eta Squared 0.26. Eta squared statistics in both subscales (0.29 and 0.26) showed a big effect and determined that there is a strong relation between pre- and post-intervention scores in two IVA+PLUS test subscales (response inhibition and attention). In addition, as data shown in Tables 6 and 7, the group received neurofeedback with cognitive rehabilitation represented more improvement as compared to the other group in two attention and impulsion factors. 


\section{Effectiveness of Neurofeedback Associated with Cognitive Rehabilitation Therapy on Children with Attention Defect Hyperactivity Disorder (ADHD)}

Table 6: multiple Comparison of interacting between variables ( 3 groups) on attention subscale

\begin{tabular}{|c|c|c|c|c|c|c|}
\hline I group & J group & $\begin{array}{c}\text { Compare means (I-J) } \\
\text { groups }\end{array}$ & $\begin{array}{c}\text { Standard } \\
\text { deviation }\end{array}$ & $\begin{array}{c}\text { Significant } \\
\text { level }\end{array}$ & \multicolumn{2}{|c|}{$\begin{array}{c}\text { confidence level } \\
95 \%\end{array}$} \\
\cline { 4 - 7 } & & & & & $\begin{array}{c}\text { Low } \\
\text { limit }\end{array}$ & up limit \\
\hline 1 & 2 & $9.760^{*}$ & 2.334 & .000 & 5.039 & 14.481 \\
& 3 & $21.938^{*}$ & 1.604 & .000 & 18.693 & 25.183 \\
\hline 2 & 1 & $-9.760^{*}$ & 2.334 & .000 & -14.481 & -5.039 \\
& 3 & $12.178^{*}$ & 1.843 & .000 & 8.451 & 15.906 \\
\hline 3 & 1 & $-21.938^{*}$ & 1.604 & .000 & -25.183 & -18.693 \\
& 2 & $-12.178^{*}$ & 1.843 & .000 & -15.906 & -8.451 \\
\hline
\end{tabular}

Groups: 1. Neurofeedback \& rehabilitation 2. Neurofeedback 3. Control

Table 7: multiple Comparison of interacting between variables (3 groups) on impulsivity subscale

\begin{tabular}{|c|c|c|c|c|c|c|}
\hline I group & J group & $\begin{array}{c}\text { Compare means (I-J) } \\
\text { groups }\end{array}$ & $\begin{array}{c}\text { Standard } \\
\text { deviation }\end{array}$ & $\begin{array}{c}\text { Significant } \\
\text { level }\end{array}$ & \multicolumn{2}{|c|}{$\begin{array}{c}\text { confidence level } \\
95 \%\end{array}$} \\
\cline { 5 - 7 } & & & & & $\begin{array}{c}\text { Low } \\
\text { limit }\end{array}$ & up limit \\
\hline 1 & 2 & $11.753^{*}$ & 2.334 & .000 & 5.039 & 14.481 \\
& 3 & $23.947^{*}$ & 1.604 & .000 & 17.693 & 25.183 \\
\hline 2 & 1 & $-8.662^{*}$ & 2.334 & .000 & -13.481 & -5.039 \\
& 3 & $11.178^{*}$ & 1.843 & .000 & 7.451 & 14.906 \\
\hline 3 & 1 & $-22.838^{*}$ & 1.604 & .000 & -25.183 & -17.693 \\
& 2 & $-11.185^{*}$ & 1.843 & .000 & -15.906 & -7.451 \\
\hline
\end{tabular}

Groups: 1. Neurofeedback \& rehabilitation 2. Neurofeedback 3. Control

\section{DISCUSSION}

The goal of this research was creating a more enriched and integrated protocol to successfully treat attention deficit and hyperactivity disorder. Based on obtained results, only neuro feedback group and neuro feedback integrated with cognitive training showed better results in comparison to control group. This result indicated the neuro feedback impact on treating children with ADHD disorder. This finding is relevant with previous studies such as Gonzeleberge et al (2009), Ahmadlou, Rostami and Sadeghi (2012) and Marizzio et al (2013) (24, 29, 30). The purpose of neurofeedback is changing brain waves with cortex self-regulatory where people learn how to focus on an issue; in addition, it is concentrated on generalizing this status into other situations outside treatment environment. That is why it can be said that children showed better results in attention factor. The combined group of neurofeedback and cognitive 


\section{Effectiveness of Neurofeedback Associated with Cognitive Rehabilitation Therapy on Children with Attention Defect Hyperactivity Disorder (ADHD)}

rehabilitation provided better results compared to the two other groups. In preceding researches, applying the cognitive training method, it was not so much practical; hence, only some executive, executive areas of brain were enabled (2). This research used cognitive rehabilitation program. As it was stated, this method focuses on working memory and executive processes subsets. Thus, improving this part of memory is the cause of this effect. As mentioned, in theta/Beta treatment with neurofeedback, children learn to decrease theta activity (4-8 HZ), and increase Beta activity. Then, this theta/beta activity indicates a neurophysiological defect. Regardless of this supposed defect, neurofeedback consider as a method to improve cognition and attention (22). While neurofeedback influences on brain waves and enhances concentration, cognitive rehabilitation works on cognitive processes involving in keeping attention on information and improves controlling. In fact, one of the difficulties of the children with this disorder is disability in concentrating on an issue, or, in other word, distraction. So, that is why response control and inhibition is too difficult in these children; then, impulsion symptoms are manifested. It may be said that rehabilitation influences on impulsion symptoms; therefore, children showed better results in response inhibition. Goals of ADHD interventions are complicated, and this is a challenge to detection of their impact on symptoms. For example, one treatment can be useful for one symptom that leads to improve quality of daily life, but we do not have any questionnaire to measure it (2). In this study, there was short interval between treatment sessions, while for some children, it is better to consider more sessions with long interval between sessions. It will lead to stability and more self-regulation and they can transfer them to the daily life easily. It is like the methods that are common in conventional cognitive behavior therapy (15). It is suggested to consider this for future studies.

\section{LIMITATIONS}

The goals of ADHD interventions are complicated and knowing which change is required to improve performance is also a big challenge. For instance, an intervention may improve one of the symptoms that may lead to a child function. Sometimes, not achieving to a certain result can be due to instrumentation. Therefore, may be the method used in this research is only effective in two attention and impulsion areas of the response inhibition. So, it is better to apply other measurement tools, too; in addition to using parents or teachers check list reports to analyze the results. Another issue is the number of samples. In this study, the number of each group were finally reduced to ten people as some limitations such as path (ten sessions commuting was difficult for families) which made difficult generalizing findings to larger samples. On the other hand, controlling children's environmental and family events was impossible during intervention. Therefore, regarding that this study used these two methods in attention deficit and hyperactivity, for the first time; it is required to do more studies with larger samples, expanded tools according to family context. 


\section{Effectiveness of Neurofeedback Associated with Cognitive Rehabilitation Therapy on Children with Attention Defect Hyperactivity Disorder (ADHD)}

\section{CONCLUSION}

Both experimental groups showed improvement in attention and response inhibition factors as compared to experimental group. The group that only received neuro feedback represented better results in IVA test by improving and increasing Beta waves. In contrast, the groups received cognitive rehabilitation beside neuro feedback, along with improving waves with working memory enhancement, also attained better scores in IVA, particularly in response inhibition factor.This study demonstrated that the effect of neuro feedback on children with ADHD can be increased by using a supplementary treatment like cognitive rehabilitation and to apply alternative and more complete mental treatments regarding drug therapy risks. This study, again, approved the role of training-based computer treatments.

\section{REFERENCES}

Biederman J, Monuteaux MC, Doyle AE, et al, (2004), Impact of executive function deficits and attention-deficit/hyperactivity disorder (ADHD) on academic outcomes in children. $\mathbf{J}$ Consult Clin Psychol.;72:757-766.

Steiner NJ1, Frenette EC, Rene KM, Brennan RT, Perrin EC.( 2014), Neurofeedback and Cognitive Attention Training for Children with Attention-Deficit Hyperactivity Disorder in Schools.

LeFever GB, Villers MS, Morrow AL, et al. Parental perceptions of adverse educational outcomes among children diagnosed and treated for ADHD: a call for improved school/provider collaboration. Psychol Schools.2002; 39:63-71.

Sergeant JA. (2005).Modeling attention-deficit/ hyperactivity disorder: a critical appraisal of the cognitive-energetic model. Biol. Psychiatry 57(11), 1248-1255.

Sonuga-Barke EJ, Castellanos FX. (2007). Spontaneous attentional fluctuations in impaired states and pathological conditions: a neurobiological hypothesis. Neurosci.Biobehav. Rev. 31(7), 977-986.

Blum, K., Chen, A. L. C., Braverman, E. R., Comings, D. E., Chen, T. J., Arcuri, V., ... \& OscarBerman, M. (2008). Attention-deficit-hyperactivity disorder and reward deficiency syndrome. Neuropsychiatric disease and treatment, 4(5), 893.

Barkley RA, Fischer M, Smallish L, Fletcher K. (2006). Young adult outcome of hyperactive children: adaptive functioning in major life activities. J. Am. Acad. Child Adolesc. Psychiatry 45(2), 192-202.

Lansbergen, M. M., van Dongen-Boomsma, M., Buitelaar, J. K., \&Slaats-Willemse, D. (2011). ADHD and EEG-neurofeedback: a double-blind randomized placebo-controlled feasibility study. Journal of Neural Transmission, 118(2), 275-284.

Gevensleben, H., Rothenberger, A., Moll, G. H., \& Heinrich, H. (2012). Neurofeedback in children with ADHD: validation and challenges

Adler LD, Nierenberg AA. (2010). Review of medication adherence in children and adults with ADHD. Postgrad Med. 122:184-191. doi: 10.3810. 


\section{Effectiveness of Neurofeedback Associated with Cognitive Rehabilitation Therapy on Children with Attention Defect Hyperactivity Disorder (ADHD)}

Charach A, Ickowicz A, Schachar R (2004) Stimulant treatment over five years: adherence, effectiveness, and adverse effects. J Am Acad Child Adolesc Psychiatry 43:559-567

Charach A, Figueroa M, Chen S, Ickowicz A, Schachar R (2006). Stimulant treatment over 5 years: effects on growth. J Am Acad Child Adolesc Psychiatry 45:415-421

Faraone SV, Biederman J, Morley CP, et al. (2008). Effect of stimulants on height and weight: a review of the literature. J Am Acad Child Adolesc Psychiatry. 47:994-1009.

Chronis AM, Jones HA, Raggi VL. (2006). Evidence-based psychosocial treatments for children and adolescents with attentiondeficit/ hyperactivity disorder.ClinPsychol Rev. 26:486.

Fabiano GA, Pelham WE, Coles EK, et al. (2009). A meta-analysis of behavioral treatments for attention-deficit/hyperactivity disorder.ClinPsychol Rev. 29:129-140.

Chronis AM, Fabiano GA, Gnagy EM, et al. (2004). An evaluation of the summer treatment program for children with attention deficit/ hyperactivity disorder using a treatment withdrawal design.BehavTher. 35:561-585.

Farmer EM, Compton SN, Burns BJ, et al. (2002). Review of the evidence base for treatment of childhood psychopathology: externalizing disorders. J Consult Clin Psychol. 70:1267.

Steiner NJ, Sheldrick RC, Gotthelf D, Perrin E. (2011). Computer-based attention training in the schools for children with attention deficit/hyperactivity disorder: A preliminary trial. ClinPediatr. 50:615-622.

Sherlin LH, Arns M, Lubar J, et al. (2011). Neurofeedback and basic learning heory: implications for research and practice. J Neurother. 2011; 15:292-304

Meisel V1, Servera M, Garcia-Banda G, Cardo E, Moreno I. (2013), Neurofeedback and standard pharmacological intervention in ADHD:A randomized controlled trial with sixmonth follow-up

Arns M, de Ridder S, Strehl U, et al. (2009). Efficacy of neurofeedback treatment in ADHD: the effects on inattention, impulsivity and hyperactivity: a meta-analysis. Clin EEG Neurosci. 2009;40: 180-189.

Klingberg T, Fernell E, Olsen PJ, et al. Computerized training of working memory in children with ADHD- A randomized, controlled trial. J Am Acad Child Adolesc Psychiatry. 2005;44: 177-186.

Shalev L, Tsal Y, Mevorach C. Computerized progressive attentional training (CPAT) program: effective direct intervention for children with ADHD. Child Neuropsychol. 2007;13:382388. doi: 10. 1080/09297040600770787.

Gevensleben H, Holl B, Albrecht B, et al. (2009). Is neurofeedback an efficacious treatment for ADHD? A randomized controlled clinical trial. J Child Psychol Psychiatry. 50:780-789. doi: 10.1111/j. 1469-7610.2008.02033.x.

Rapport, M. D., Alderson, M.R., Kofler, M. J., Sarver, D.E., Bolden, J \&Sims ,V., (2008). Working Memory Deficits in Boys with Attention-deficit/ Hyperactivity Disorder (ADHD): The Contribution of Central Executive and Subsystem Processes.Journal ofAbnormal Child Psychology, 36:825-837. 
Barkley, R. A. (2006). Attention-deficit hyperactivity disorder: A handbook for diagnosis and treatment( 3 rh ed.). New York: Guilford Press.

Mashhadi, M. Teymouri, S. Soltanifar, A. Hoseinzademaleki, Z. (2011). Visuo-spatial working memory of ADHD children in Benton Visual Retention Test. 1th conferences of cognitive science in education.848-857.

Nejati, V. Bahrami, H. Abrovan, M. roubinzade, SH. Motiei, H. (1391). Executive functions and working memory in children with attention defect hyperactivity disorder. Journal of medical science, Gorgan, 3: 69-76.

Ahmadloua, Mehran, Rostami, Reza; VahidSadeghi, (2012), which attentiondeficit/hyperactivity disorder children will be improved through neurofeedback therapy? A graph theoretical approach to neocortex neuronal network of ADHD.Neuroscience Letters 516. $156-160$.

Maurizio, S. Liechti, M. D. Brandeis, D. Jacke, L. Drechsler, R. (2013). Differential EMG Biofeedback for Children with ADHD: A Control Method for Neurofeedback Training with a Case Illustration. ApplPsychophysiol Biofeedback.DOI 10.1007/s10484-013-9213$\mathrm{x}$.

Heinrich H, Gevensleben H, Strehl U (2007) Annotation: neurofeedback - train your brain to train behaviour. J Child Psychol Psychiatry 48:3-16. 